\title{
3-(2-氯-4-三氟甲基)苯氧基取代苯甲酰腙衍生物的合成与生物活性
}

\author{
刘建超 崔泽平 贺红武* \\ (华中师范大学化学学院 武汉 430079)
}

\begin{abstract}
摘要 选择 3-(2-氯-4-三氟甲基苯氧基)苯甲酸为起始原料, 经酰氯化，再与乙醇反应成酯，然后和水合肼反应，最后与 芳香醛缩合，合成了 10 个未见文献报道的 3-(2-氯-4-三氟甲基苯氧基)苯甲酰腙 4. 通过 IR, ${ }^{1} \mathrm{H}$ NMR, EI-MS, 元素分析 等方法对所合成的化合物进行了结构表征. 代表化合物 4-氯苯亚甲基-3-(2-氯-4-三氟甲基苯氧基)苯甲酰腙(4f)经单晶 X 衍射证实了结构. 并初步测定了所合成化合物的杀菌和除草活性. 结果表明: 部分测试化合物在 $50 \mathrm{mg} / \mathrm{L}$ 浓度下对水稻 纹枯菌和黄瓜灰霉菌具有优良的抑制效果，在 $100 \mathrm{mg} / \mathrm{L}$ 浓度下对油菜和稗草的根显示一定的抑制效果.
\end{abstract}

关键词＼cjkstart苯甲醛; 苯甲酰腙; 杀菌活性; 除草活性

\section{Synthesis and Pesticidal Activity of 3-(2-Chloro-4-trifluoromethyl)phenoxy Benzoylhydrazones}

\author{
Liu, Jianchao Cui, Zeping He, Hongwu* \\ (College of Chemistry, Central China Normal University, Wuhan 430079)
}

\begin{abstract}
A series of new substituted benzaldehyde (or 2-furaldehyde) 3-(2-chloro-4-trifluoromethyl)phenoxy benzoylhydrazones 4 have been designed and synthesized by the reactions of substituted aldehydes with intermediate 3 in $64 \% \sim 89 \%$ yields. The structures of compounds 4 have been confirmed by ${ }^{1} \mathrm{H}$ NMR, IR, EI-MS and elemental analyses. The structure of 4-chloro-benzaldehyde 3-(2-chloro-4-trifluoromethyl)phenoxy benzoylhydrazone (4f) has been determined by X-ray single crystal diffraction. The results of preliminary bioassay indicated that some compounds possess good fungicidal activities against Rhizoctonia solani and Botrytis cinereapers at a dosage of $50 \mathrm{mg} / \mathrm{L}$ and moderate herbicidal activity against the roots of rape and barnyard grass at $100 \mathrm{mg} / \mathrm{L}$.
\end{abstract}

Keywords benzaldehyde; benzoylhydrazone; fungicidal activity; herbicidal activity

酰腙化合物具有优良的生物活性、较低的毒性、强 的配位能力、很好的亲脂性, 能与生物体细胞中的金属 离子形成稳定的配合物, 从而可以影响相关的酶促反 应, 具有很好的研究前景 ${ }^{[1 \sim 4]}$. 近二十多年来, 大量文 献报道的酰腙化合物的药理活性以及作为配体的应用， 而有关酰腙化合物的农药活性的研究与应用相对较 少 ${ }^{[5,6]}$. 二芳基醚具有能显著改善农药的理化性质, 如提 高生物活性、提高稳定性、降低毒性、扩大生物活性谱 等特点, 在新农药创制中具有重要作用 ${ }^{[7]}$. 由于氟原子 的特殊性质, 含氟基团的引入有时可使化合物的生物活 性增加而毒性降低, 含氟农药的开发已成为新农药创制 研究的热点 ${ }^{[8]}$. 为了进一步探索酰腙类衍生物以及二苯 醚类化合物作为农药的生物活性, 根据生物活性叠加原
理，将上述两类化合物的核心结构进行拼接和修饰，同 时引入三氟甲基，达到研制出抗菌和除草活性更高、谱 更广而且毒性较低的农药先导化合物的目的. 本文选择 3-(2-氯-4-三氟甲基苯氧基)苯甲酸为起始原料, 经酰氯 化，再与乙醇反应成酯，然后和水合肼反应，最后与芳 香醛缩合, 合成了 10 个未见文献报道的酰腙化合物, 并 初步测定了所合成化合物的杀菌和除草活性. 其合成路 线如(Scheme 1).

\section{1 结果与讨论}

\section{1 合成方法}

本实验是在无溶剂条件下, 采用过量的二氯亚砜和

*E-mail: he1208@mail.ccnu.edu.cn; liujc@mail.ccnu.edu.cn

Received April 20, 2012; revised May 24, 2012; published online June 5, 2012.

Project supported by the National Basic Research Program of China (No. 2010CB126100), and the National Natural Science Foundation of China (Nos. 20372023, 20772042).

国家重点基础研究发展规划(973 计划) (No. 2010CB126100)、国家自然科学基金(Nos. 20372023, 20772042)资助项目. 
<smiles>CCOC(=O)c1cccc(Oc2ccc(C(=O)OCC(C)(F)F)cc2Cl)c1</smiles><smiles>[R]C=NNC(=O)c1cccc(Oc2ccc(C(F)(F)F)cc2Cl)c1</smiles>

3

4

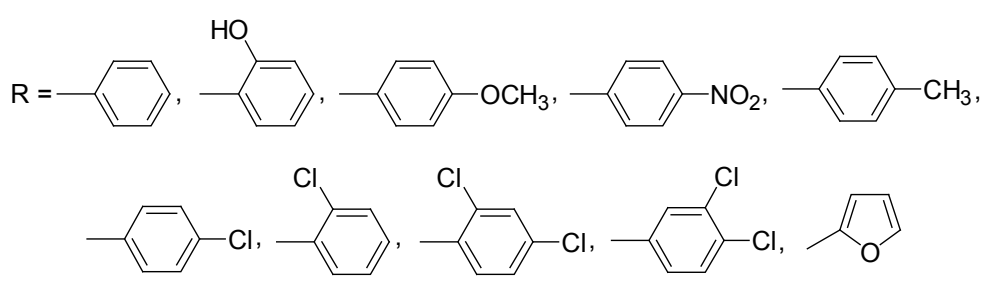

Scheme 1

取代苯甲酸在低温下反应制备中间体 $\mathbf{1}$, 实验时用 DMF $2 \sim 3$ 滴作催化剂, $40 \sim 60{ }^{\circ} \mathrm{C}$ 反应 $4 \sim 6 \mathrm{~h}$, 然后水洜减压 蒸去过量的二氯亚砜和其它反应副产物. 温度对反应的 收率有较大影响, 随反应温度不断升高, 其反应收率不 断下降. 另外, 酰氯非常活泼, 不宜久置, 现制现用. 中 间体 2 由 3-(2-氯-4-三氟甲基苯氧基)苯甲酰氯与乙醇室 温下反应制取，此反应剧烈放热，酰氯宜在冰水浴存在 下逐滴加入. 此反应也可直接由原料酸与乙醇反应来制 取, 只是纯化更困难. 中间体 $\mathbf{3}$ 的制备过程中采用了无 水乙醇作溶剂, 并使反应物水合肼大大过量的方法. 既 简化了后处理, 又缩短了反应时间, 提高了产率. 生成 目标化合物的反应可在加热回流的条件下进行, 酰肼完 全溶解后, 再搅拌下滴加相应的醛. 大部分目标产物具 有较好的溶解性, 能在二氯甲烷、丙酩、乙醇、DMSO 等溶剂中重结晶.

\section{2 结构表征}

所合成的化合物都经 IR, ${ }^{1} \mathrm{H}$ NMR, EI-MS 分析确证. 目标化合物的 ${ }^{1} \mathrm{H}$ NMR 中, ArH 的化学位移在 $\delta 6.88 \sim$ 8.04 范围内, $=\mathrm{CH}$ 在 $\delta 8.5$ 左右, $\mathrm{NH} \delta 9.8$ 在左右. 从 IR 谱图数据可看出所有特征基团均有明显的吸收峰: $\mathrm{N}$ $\mathrm{H}$ 在 $3200 \mathrm{~cm}^{-1}$ 左右有较强的吸收峰, 苯环上尖而弱的 $\mathrm{C}-\mathrm{H}$ 伸缩振动吸收带在 $3000 \mathrm{~cm}^{-1}$ 左右, 羰基 $(\mathrm{C}=\mathrm{O})$ 在 $1640 \mathrm{~cm}^{-1}$ 附近表现出强伸缩振动吸收峰, 比通常的 $\mathrm{C}=\mathrm{O}$ 振动频率明显红移, 表明所合成的化合物存在较 大的共轭体系, 导致 $\mathrm{C}=\mathrm{O}$ 键级减弱, 使 $\mathrm{C}=\mathrm{O}$ 向低波数 移动. MS 数据中可以看出所有的化合物均有很强的分 子离子峰; 在元素分析数据中理论值与计算值也符合得 很好, 其相对误差基本在 $0.5 \%$ 范围内.

\section{3 化合物的生物活性}

将目标化合物 4 配制成浓度为 $5.0 \times 10^{-5} \mathrm{~g} / \mathrm{mL}$ 药液,
采用离体平皿法对棉花枯萎菌(Fusarium oxyporium), 水稻纹枯菌(Rhizoctonia solani), 黄瓜灰霉菌(Botrytis cinereapers), 小麦赤霉菌(Gibberella zeae), 苹果轮纹菌 (Dothiorella gregaria), 棉花炭疽菌 (Colletotrichum gossypii)等 6 种菌进行了初步杀菌活性测试, 具体测试 方法见文献 $[9,10]$ ，测试结果见表 1. 将目标化合物 4 配 制成浓度为 $1.0 \times 10^{-5}$ 和 $1.0 \times 10^{-4} \mathrm{~g} / \mathrm{mL}$ 的药液, 采用表 面皿法对稗草(Barnyard grass)和油菜(Rape)的根/茎进行 了初步的除草活性测试，其测试结果见表 2 .

上述生测结果表明: 此类化合物对以上 6 种菌均具 有优良的杀菌活性, 其中对水稻纹枯菌和黄瓜灰霉菌的 抑菌效果尤为显著. 例如化合物 $\mathbf{4 j}$ 对水稻纹枯菌和黄 瓜灰需菌的抑菌率均达到了 $100 \%$; 化合物 $\mathbf{4 g}$ 对 6 种菌 都有显著的杀菌活性，抑制率在 $95 \%$ 以上，显示了良好 的广谱抑菌效果. 从表 2 可以看出, 此类化合物在 $1.0 \times 10^{-4} \mathrm{~g} / \mathrm{mL}$ 浓度下, 对油菜和稗草的根与茎表现出 一定的除草效果，并且对根的抑制活性高于对茎的抑制 活性. 如化合物 $4 \mathbf{d}$ 在该浓度下对油菜根/茎具有较好的 抑制活性 $(90.1 \% / 80.0 \%)$. 当药液浓度降为 $1.0 \times 10^{-5}$ $\mathrm{g} / \mathrm{mL}$ 时, 对油菜和稗草根茎的抑制率有所下降, 效果均 不明显.

\section{2 结论}

本文根据生物活性叠加原理, 选择 3-(2-氯-4-三氟 甲基苯氧基)苯甲酸为起始原料, 经酰氯化, 再与乙醇 反应成酯，然后和水合肼反应，最后与芳香醛缩合，合 成了 10 个未见文献报道的酰腙化合物 4. 通过 IR, ${ }^{1} \mathrm{H}$ NMR, EI-MS, 元素分析等方法对所合成的化合物进行 结构表征.

初步的杀菌和除草活性测试结果表明: 此类化合物 
表 1 目标化合物 $\mathbf{4}$ 的抑菌活性数据 $\left(5.0 \times 10^{-5} \mathrm{~g} / \mathrm{mL}\right)$

Table 1 Fungicidal activity of compounds $4\left(5.0 \times 10^{-5} \mathrm{~g} / \mathrm{mL}\right)$

\begin{tabular}{ccccccc}
\hline \multirow{2}{*}{ Compd. } & \multicolumn{5}{c}{ Inhibition rate/\% } \\
\cline { 2 - 7 } & Fusarium oxysporium & Rhizoctonia solani & Botrytis & cinereapers & Gibberella zeae & Dothiorella gregaria Colletotrichum gossypii \\
\hline $\mathbf{4 a}$ & 50.00 & 90.59 & 100 & 70.37 & 94.12 & 84.62 \\
$\mathbf{4 b}$ & 77.27 & 80.46 & 82.61 & 77.42 & 84.62 & 59.09 \\
$\mathbf{4 c}$ & 77.27 & 86.96 & 98.15 & 87.50 & 88.24 & 84.48 \\
$\mathbf{4 d}$ & 45.45 & 88.51 & 93.48 & 54.84 & 53.85 & 45.45 \\
$\mathbf{4 e}$ & 59.09 & 89.66 & 95.65 & 87.10 & 69.23 & 68.18 \\
$\mathbf{4 f}$ & 78.18 & 93.10 & 95.65 & 70.97 & 76.92 & 81.82 \\
$\mathbf{4 g}$ & 95.45 & 98.37 & 100 & 100 & 100 & 100 \\
$\mathbf{4 h}$ & 81.82 & 97.70 & 100 & 93.55 & 96.15 & 86.36 \\
$\mathbf{4 i}$ & 59.09 & 91.95 & 95.65 & 80.65 & 76.92 & 72.73 \\
$\mathbf{4 j}$ & 68.78 & 100 & 100 & 96.43 & 94.12 & 83.10 \\
\hline
\end{tabular}

${ }^{a}$ 此表数据由华中师范大学化学学院生测室提供.

表 2 目标化合物 4 对油菜和稗草抑制活性 ${ }^{a}$

Table 2 Herbicidal activity of compounds 4

\begin{tabular}{|c|c|c|c|c|}
\hline \multirow{3}{*}{ Compd. } & \multicolumn{4}{|c|}{ Relative inhibition (root/stalk, \%) } \\
\hline & \multicolumn{2}{|c|}{ Rape } & \multicolumn{2}{|c|}{ Barnyard grass } \\
\hline & $1.0 \times 10^{-4} \mathrm{~g} / \mathrm{mL}$ & $1.0 \times 10^{-5} \mathrm{~g} / \mathrm{mL}$ & $1.0 \times 10^{-4} \mathrm{~g} / \mathrm{mL}$ & $1.0 \times 10^{-5} \mathrm{~g} / \mathrm{mL}$ \\
\hline $4 \mathbf{a}$ & $63.8 / 4.2$ & $23.1 /-38.3$ & $40.3 /-9.3$ & $-23.2 /-14.8$ \\
\hline $4 b$ & $76.3 / 60.9$ & $8.5 /-8.7$ & $84.2 / 53.3$ & $73.7 / 40.0$ \\
\hline $4 c$ & $85.9 / 35.8$ & $29.2 /-29.1$ & $86.2 / 15.3$ & $3.3 /-9.3$ \\
\hline 4d & $90.1 / 80.0$ & $19.7 / 31.4$ & $94.4 / 66.7$ & $36.1 / 16.7$ \\
\hline $4 e$ & $79.7 / 78.3$ & $30.5 / 30.4$ & $78.9 / 33.3$ & $57.9 / 26.7$ \\
\hline $4 f$ & $76.3 / 56.5$ & $37.3 / 39.1$ & $57.9 / 13.3$ & $52.6 / 0$ \\
\hline $4 g$ & $90.4 / 49.2$ & $35.4 /-7.5$ & $76.2 / 8.2$ & $8.8 /-3.1$ \\
\hline $4 \mathrm{~h}$ & $91.5 / 69.6$ & $37.3 / 26.1$ & $78.9 / 40.0$ & $68.4 / 26.7$ \\
\hline $4 \mathbf{i}$ & $81.4 / 69.6$ & $57.6 / 52.2$ & $84.2 / 26.7$ & $73.7 / 53.3$ \\
\hline $4 \mathbf{j}$ & $86.7 / 6.1$ & $46.2 /-25.0$ & $88.9 / 51.9$ & $35.9 / 14.7$ \\
\hline
\end{tabular}

${ }^{a}$ 此表数据由华中师范大学化学学院生测室提供.

对棉花枯萎菌、水稻纹枯菌、黄瓜灰霉菌、小麦赤霉菌、 苹果轮纹菌、棉花炭疽菌等 6 种菌均具有优良的杀菌活 性, 其中对水稻纹枯菌和黄瓜灰霉菌的抑菌效果尤为显 著. 在 $1.0 \times 10^{-4} \mathrm{~g} / \mathrm{mL}$ 浓度下, 该类化合物对油菜和稗 草的根与茎表现出一定的除草效果, 并且对根的抑制活 性高于对茎的抑制活性; 当药液浓度降为 $1.0 \times 10^{-5}$ $\mathrm{g} / \mathrm{mL}$ 时, 对油菜和稗草根茎的抑制率有所下降, 效果均 不明显.

\section{3 实验部分}

\section{1 仪器与试剂}

红外光谱用 AVATAR360 型傅立叶红外光谱仪 $\left(\mathrm{KBr}\right.$ 压片); ${ }^{1} \mathrm{H}$ NMR 用 Varian XL-400 型核磁光谱仪(以 TMS 为内标, 以 $\mathrm{CDCl}_{3}$ 为溶剂); 质谱用 Finnigen TRA CE 型质谱仪; LC-MS 采用 API200 型液质联用仪; 元素 分析采用 VarioE L111 CHN 元素分析仪; 熔点采用上海 精密科学仪器有限公司生产的 WRS-IB 型熔点仪测定 (温度未经校正)或北京第三光学仪器厂 $\mathrm{X} 4$ 型熔点仪. 二氯甲烷, 无水乙醇, 水合肼 $(85 \%)$, 碳酸氢钠, 芳香醛,
乙醚, DMF 均为分析纯, 二氯亚砜使用前重蒸.

3.2 中间体 3-(2-氯-4-三氟甲基苯氧基)苯甲酰氯(1), 3-(2-氯-4-三氟甲基苯氧基)苯甲酸乙酯(2)和 3-(2-氯-4三氟甲基苯氧基)苯甲酰肼(3)的制备

中间体 1 3 的制备参照文献[11]方法. 3-(2-氯-4-三 氟甲基苯氧基)苯甲酰肼(3)的收率为 72\%，白色固体. m.p. $116 \sim 117{ }^{\circ} \mathrm{C}$; EI-MS $(70 \mathrm{eV}) \mathrm{m} / z(\%): 331\left(\mathrm{M}^{+}\right.$, 100). Anal. calcd for $\mathrm{C}_{14} \mathrm{H}_{10} \mathrm{ClF}_{3} \mathrm{~N}_{2} \mathrm{O}_{2}: \mathrm{C} 50.85, \mathrm{H} 3.05, \mathrm{~N}$ 8.47; found C 51.23, H 2.89, N 8.26.

\subsection{3-(2-氯-4-三氟甲基苯氧基)苯甲酰腙 4 的制备}

以苯亚甲基-3-(2-氯-4-三氟甲基苯氧基)苯甲酰腙 $4 \mathbf{a}$ 的制备为例. 在 $100 \mathrm{~mL}$ 三颈烧瓶中, 加入 3-(2-氯-4三氟甲基苯氧基)苯甲酰肼 $3.3 \mathrm{~g}(0.01 \mathrm{mmol})$, 苯甲醛 $1.1 \mathrm{~g}(0.01 \mathrm{mmol}), 30 \mathrm{~mL}$ 无水乙醇, 回流 $4 \mathrm{~h}$, 冷却后加 入蒸馏水，析出沉淀，抽滤，滤饼依次用水，无水乙醇， 无水乙醚洗涤，红外灯下干燥，用 $95 \%$ 乙醇重结晶，得 白色固体, 产率 $70 \%$. m.p. $146 \sim 147{ }^{\circ} \mathrm{C}$. 参照上述方法 可以合成目标化合物 $\mathbf{4 b \sim 4 j}$.

苯亚甲基-3-(2-氯-4-三氟甲基苯氧基)苯甲酰腙(4a): 
产率 70\%，白色固体. m.p. $146 \sim 147{ }^{\circ} \mathrm{C} ;{ }^{1} \mathrm{H}$ NMR $\left(\mathrm{CDCl}_{3}\right) \delta: 7.03 \sim 7.84(\mathrm{~m}, 12 \mathrm{H}, \mathrm{ArH}), 8.48(\mathrm{~s}, 1 \mathrm{H}, \mathrm{N}=$ $\mathrm{CH}), 10.25$ (s, 1H, NH); IR (KBr) v: 3197, 3066, 2852, $1647(\mathrm{C}=\mathrm{O}), 1579,1559,1498,1438,1408,1326,1268$, 1168, 1123, 1083, 1060, $897 \mathrm{~cm}^{-1}$; EI-MS $(70 \mathrm{eV}) \mathrm{m} / \mathrm{z}$ (\%): $419\left(\mathrm{M}^{+}, 100\right)$. Anal. calcd for $\mathrm{C}_{21} \mathrm{H}_{14} \mathrm{ClF}_{3} \mathrm{~N}_{2} \mathrm{O}_{2}$ : C $60.23, \mathrm{H} 3.73$, N 6.69; found C 60.50, H 3.59, N 6.69.

2-羟基苯亚甲基-3-(2-氯-4-三氟甲基苯氧基)苯甲酰 腙 $(4 b)$ : 产率 $68 \%$, 白色固体. m.p. $149 \sim 150{ }^{\circ} \mathrm{C} ;{ }^{1} \mathrm{H}$ NMR $\left(\mathrm{CDCl}_{3}\right) \delta: 6.88 \sim 7.74(\mathrm{~m}, 11 \mathrm{H}, \mathrm{ArH}), 8.49(\mathrm{~s}, 1 \mathrm{H}$, $\mathrm{N}=\mathrm{CH}), 9.44$ (s, 1H, NH), 11.01 (s, 1H, OH); IR (KBr) $v$ : 3440, 3214, 3057, $1648(\mathrm{C}=\mathrm{O}), 1618,1581,1562,1490$, 1399, 1326, 1268, 1167, 1081, $891 \mathrm{~cm}^{-1}$; EI-MS $(70 \mathrm{eV})$ $m / z$ (\%): $435\left(\mathrm{M}^{+}, 100\right)$. Anal. calcd for $\mathrm{C}_{21} \mathrm{H}_{14} \mathrm{ClF}_{3} \mathrm{~N}_{2} \mathrm{O}_{3}$ : C 58.01, H 3.25, N 6.44; found C 58.53, H 3.22, N 6.39.

4-甲氧基苯亚甲基-3-(2-氯-4-三氟甲基苯氧基)苯甲 酰腙(4c): 产率 $64 \%$, 白色固体. m.p. 164 165 ${ }^{\circ} \mathrm{C} ;{ }^{1} \mathrm{H}$ $\operatorname{NMR}\left(\mathrm{CDCl}_{3}\right) \delta: 3.87\left(\mathrm{~s}, 3 \mathrm{H}, \mathrm{OCH}_{3}\right), 6.91 \sim 7.86(\mathrm{~m}, 11 \mathrm{H}$, ArH), 8.24 (s, $1 \mathrm{H}, \mathrm{N}=\mathrm{CH}), 9.89$ (s, 1H, NH); IR (KBr) $v$ : 3192, 3051, 2840, $1646(\mathrm{C}=\mathrm{O}), 1588,1514,1498,1408$, 1368, 1257, 1163, 1082, $830 \mathrm{~cm}^{-1}$; EI-MS $(70 \mathrm{eV}) \mathrm{m} / \mathrm{z}$ (\%): $449\left(\mathrm{M}^{+}, 100\right)$. Anal. calcd for $\mathrm{C}_{22} \mathrm{H}_{16} \mathrm{ClF}_{3} \mathrm{~N}_{2} \mathrm{O}_{3}$ : C 58.87, H 3.59, N 6.24; found C 58.62, H 3.73, N 6.30.

4-硝基苯亚甲基-3-(2-氯-4-三氟甲基苯氧基)苯甲酰 腙(4d): 产率 65\%, 白色固体. m.p. $185 \sim 187{ }^{\circ} \mathrm{C} ;{ }^{1} \mathrm{H}$ NMR $\left(\mathrm{CDCl}_{3}\right) \delta: 7.05 \sim 7.76(\mathrm{~m}, 11 \mathrm{H}, \mathrm{ArH}), 8.26(\mathrm{~s}, 1 \mathrm{H}$, $\mathrm{N}=\mathrm{CH}), 9.42(\mathrm{~s}, 1 \mathrm{H}, \mathrm{NH})$; IR $(\mathrm{KBr}) v: 3192,2986,2857$, $1652(\mathrm{C}=\mathrm{O}), 1581,1543,1508,1476,1320,1268,1169$, 894, $829 \mathrm{~cm}^{-1}$; EI-MS (70 eV) m/z (\%): $464\left(\mathrm{M}^{+}, 37\right)$. Anal. calcd for $\mathrm{C}_{21} \mathrm{H}_{13} \mathrm{ClF}_{3} \mathrm{~N}_{3} \mathrm{O}_{4}$ : C 54.38, H 2.83, N 9.06; found C 54.97, H 2.63, N 8.88.

4-甲基苯亚甲基-3-(2-氯-4-三氟甲基苯氧基)苯甲酰 腙 (4e): 产率 65\%, 白色固体. m.p. $175 \sim 176{ }^{\circ} \mathrm{C} ;{ }^{1} \mathrm{H}$ NMR $\left(\mathrm{CDCl}_{3}\right) \delta: 2.41\left(\mathrm{~s}, 3 \mathrm{H}, \mathrm{CH}_{3}\right), 7.02 \sim 7.79(\mathrm{~m}, 11 \mathrm{H}$, ArH), 8.26 (s, $1 \mathrm{H}, \mathrm{N}=\mathrm{CH}), 9.49$ (s, 1H, NH); IR (KBr) v: 3182, 3057, $1648(\mathrm{C}=\mathrm{O}), 1581,1545,1490,1399,1363$, 1300, 1268, 1167, 1081, $891 \mathrm{~cm}^{-1}$; EI-MS (70 eV) $\mathrm{m} / \mathrm{z}$ (\%): $433\left(\mathrm{M}^{+}, 100\right)$. Anal. calcd for $\mathrm{C}_{22} \mathrm{H}_{16} \mathrm{ClF}_{3} \mathrm{~N}_{2} \mathrm{O}_{2}$ : C 61.05, H 3.73, N 6.47; found C 60.93, H 3.83, N 6.56.

4-氯苯亚甲基-3-(2-氯-4-三氟甲基苯氧基)苯甲酰腙 (4f): 产率 89\%, 白色固体. m.p. $175 \sim 177{ }^{\circ} \mathrm{C} ;{ }^{1} \mathrm{H}$ NMR $\left(\mathrm{CDCl}_{3}\right) \delta: 6.99 \sim 7.84(\mathrm{~m}, 11 \mathrm{H}, \mathrm{ArH}), 8.32(\mathrm{~s}, 1 \mathrm{H}, \mathrm{N}=$ $\mathrm{CH}$ ), 9.89 (s, 1H, NH ); IR (KBr) v: 3148, 3058, 1640 $(\mathrm{C}=\mathrm{O}), 1580,1562,1491,1440,1403,1301,1288,1128$, $893 \mathrm{~cm}^{-1}$; EI-MS $(70 \mathrm{eV}) \mathrm{m} / z(\%): 453\left(\mathrm{M}^{+}, 90\right)$. Anal. calcd for $\mathrm{C}_{21} \mathrm{H}_{13} \mathrm{Cl}_{2} \mathrm{~F}_{3} \mathrm{~N}_{2} \mathrm{O}_{2}$ : C 55.65, H 2.89, $\mathrm{N}$ 6.18; found C 55.32, H 2.92, N 6.41.

2-氯苯亚甲基-3-(2-氯-4-三氟甲基苯氧基)苯甲酰腙 (4g): 产率 68\%, 白色固体. m.p. 139 $141{ }^{\circ} \mathrm{C} ;{ }^{1} \mathrm{H}$ NMR $\left(\mathrm{CDCl}_{3}\right) \delta: 7.26 \sim 8.04(\mathrm{~m}, 11 \mathrm{H}, \mathrm{ArH}), 8.05(\mathrm{~s}, 1 \mathrm{H}, \mathrm{N}=$ $\mathrm{CH}), 10.50$ (s, 1H, NH); IR (KBr) v: 3178, 3014, 1661 $(\mathrm{C}=\mathrm{O}), 1581,1560,1500,1336,1301,1179,1031,815$ $\mathrm{cm}^{-1}$; EI-MS (70 eV) $\mathrm{m} / z(\%): 453\left(\mathrm{M}^{+}, 70\right)$. Anal. calcd for $\mathrm{C}_{21} \mathrm{H}_{13} \mathrm{Cl}_{2} \mathrm{~F}_{3} \mathrm{~N}_{2} \mathrm{O}_{2}$ : C 55.65, H 2.89, N 6.18; found $\mathrm{C}$ $55.23, \mathrm{H} 2.68, \mathrm{~N} 6.16$.

2,4-二氯苯亚甲基-3-(2-氯-4-三氟甲基苯氧基)苯甲 酰腙(4h): 产率 67\%，白色固体. m.p. 186 188 ${ }^{\circ} \mathrm{C} ;{ }^{1} \mathrm{H}$ NMR $\left(\mathrm{CDCl}_{3}\right) \delta: 7.01 \sim 7.75(\mathrm{~m}, 10 \mathrm{H}, \operatorname{ArH}), 8.63(\mathrm{~s}, 1 \mathrm{H}$, $\mathrm{N}=\mathrm{CH}), 9.74(\mathrm{~s}, 1 \mathrm{H}, \mathrm{NH}) ; \mathrm{IR}(\mathrm{KBr}) v: 3197,3066,1639$ $(\mathrm{C}=\mathrm{O}), 1561,1499,1439,1367,1302,1172,1080,890$ $\mathrm{cm}^{-1}$; EI-MS $(70 \mathrm{eV}) \mathrm{m} / \mathrm{z}(\%): 488\left(\mathrm{M}^{+}, 100\right)$. Anal. calcd for $\mathrm{C}_{21} \mathrm{H}_{12} \mathrm{Cl}_{3} \mathrm{~F}_{3} \mathrm{~N}_{2} \mathrm{O}_{2}$ : C 51.72, H 2.48, N 5.74; found $\mathrm{C}$ $51.90, \mathrm{H} 2.57$, N 5.65.

3,4-二氯苯亚甲基-3-(2-氯-4-三氟甲基苯氧基)苯甲 酰腙(4i): 产率 $63 \%$ ，白色固体. m.p. $163 \sim 165{ }^{\circ} \mathrm{C} ;{ }^{1} \mathrm{H}$ $\operatorname{NMR}\left(\mathrm{CDCl}_{3}\right) \delta: 7.01 \sim 7.77(\mathrm{~m}, 10 \mathrm{H}, \mathrm{ArH}), 8.33(\mathrm{~s}, 1 \mathrm{H}$, $\mathrm{N}=\mathrm{CH}), 9.73$ (s, 1H, NH); IR (KBr) v: 3192, 2965, 1648 $(\mathrm{C}=\mathrm{O}), 1561,1508,1475,1304,1288,1125,1080,894$ $\mathrm{cm}^{-1}$; EI-MS (70 eV) $\mathrm{m} / z(\%): 488\left(\mathrm{M}^{+}, 87\right)$. Anal. calcd for $\mathrm{C}_{21} \mathrm{H}_{12} \mathrm{Cl}_{3} \mathrm{~F}_{3} \mathrm{~N}_{2} \mathrm{O}_{2}$ : C 51.72, H 2.48, N 5.74; found $\mathrm{C}$ $51.44, \mathrm{H} 2.39$, N 5.85 .

2-呋喃亚甲基-3-(2-氯-4-三氟甲基苯氧基)苯甲酰腙 (4j): 产率 78\%, 白色固体. m.p. $165 \sim 166{ }^{\circ} \mathrm{C} ;{ }^{1} \mathrm{H}$ NMR $\left(\mathrm{CDCl}_{3}\right) \delta: 6.07 \sim 6.55(\mathrm{~m}, 3 \mathrm{H}$, furanyl-H), $7.13 \sim 7.77(\mathrm{~m}$, $7 \mathrm{H}, \mathrm{ArH}), 8.48(\mathrm{~s}, 1 \mathrm{H}, \mathrm{N}=\mathrm{CH}), 11.24(\mathrm{~s}, 1 \mathrm{H}, \mathrm{NH})$; IR (KBr) v: 3193, 3064, $1651(\mathrm{C}=\mathrm{O}), 1562,1326,1301$, 1268, 1170, $1083 \mathrm{~cm}^{-1}$; EI-MS (70 eV) $\mathrm{m} / z(\%): 409\left(\mathrm{M}^{+}\right.$, 75). Anal. calcd for $\mathrm{C}_{19} \mathrm{H}_{12} \mathrm{ClF}_{3} \mathrm{~N}_{2} \mathrm{O}_{3}$ : C 55.83, H 2.96, N 6.85; found C 55.45, H 3.03, N 6.86.

\section{$3.44 \mathrm{f}$ 的晶体结构测定}

为了对该系列化合物分子结构作进一步的验证，从 无水乙醇中培养了化合物 $4 \mathbf{f}$ 单晶用于 $\mathrm{X}$ 射线单晶衍射 实验. 使用 Smart Apex CCD 衍射仪, 用经过石墨单色 器单色化 $\mathrm{Mo} \mathrm{K \alpha}$ 的射线 $(\lambda=0.71073 \AA)$ 作为入射辐射. 在 $1.84^{\circ} \leqslant \theta \leqslant 27^{\circ}$ 范围内, 以 $\omega-2 \theta$ 扫描方式, 在室温 $(19 \pm 2){ }^{\circ} \mathrm{C}$ 下共收集到场 14666 个独立反射点, 其中 4363 个为可观测反射 $[I \geqslant 2 \sigma(I)], R_{\text {int }}=0.0316$, 全部强度 数据均用 SAD ABS 程序校正. 该晶体属单斜晶系, 空 间群为 $P 2(1) / c, a=18.8997(18) \AA, b=13.7107(13) \AA$, $c=7.8645(8) \AA ; \beta=98.573(2)^{\circ}, V=2015.1(3) \AA^{3}, M_{\mathrm{r}}=$ 
453.23, $Z=4, D_{\mathrm{c}}=1.494 \mathrm{Mg} / \mathrm{m}^{3}, \mu=0.370 \mathrm{~mm}^{-1}, R=$ CCDC 号为 882127. 4f 的分子结构见图 1.

$0.0581, w R=0.1524$. 数据存于英国剑桥数据中心,

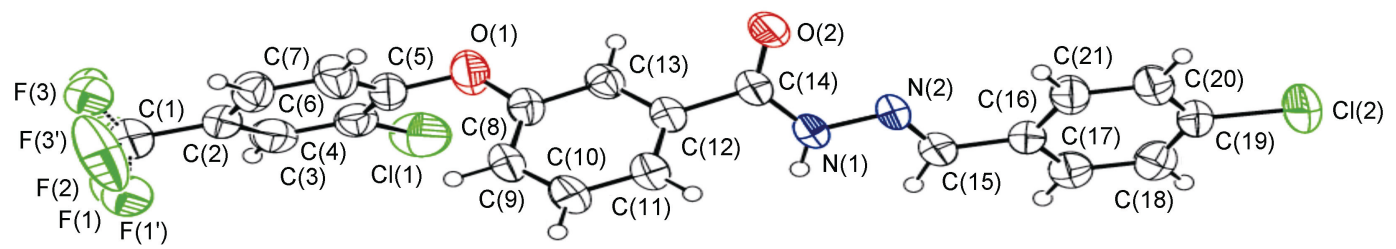

图 $14 \mathrm{f}$ 的分子结构图

Figure 1 Molecular structure of $\mathbf{4 f}$

\section{References}

[1] Singh, V. P.; Katiyar, A. Pestic. Biochem. Physiol. 2008, 92, 8.

[2] Ni, Z.; Xue, S.; Wang, J.; Meng, W. Chin. J. Org. Chem. 2011, 32, 222 (in Chinese). (倪振杰, 薛思佳, 王静, 孟雯, 有机化学, 2011, 32, 222.)

[3] Ling, A. L.; Hong, Y.; Gonzalez, J.; Gregor, V.; Polinsky, A.; Kuki, A.; Shi, S.; Teston, K.; Murphy, D.; Porter, J.; Kiel, D.; Lakis, J.; Anderes, K.; May, J.; Knudsen, L. B.; Jesper. L. J. Med. Chem. 2001, 44, 3141.

[4] Ling, A.; Plewe, M.; Gonzalez, J.; Madsen, P.; Sams, C. K.; Lau, J.; Gregor, V.; Murphy, D.; Teston, K.; Kuki, A.; Shi, S.; Truesdale, L.; Kiel, D.; May, J.; Lakis, J.; Anderes, K.; Iatsimirskaia, E.; Sidelmann, U. G.; Knudsen, L. B.; Btand, C. L.; Polinsky, A. Bioorg. Med. Chem. Lett. 2002, 12, 663.

[5] Bernhardt, P. V.; Mattsson, J.; Richardson, D. R. Inorg. Chem. 2006, 45, 752 .
[6] Kalinowski, D. S.; Sharpe, P. C.; Bernhardt, P. V.; Richardson, D. R. J. Med. Chem. 2008, 51, 331.

[7] Wang, M. H.; Yang, C. L.; Jiang, M. G. World Pestic. 2002, 24, 13 (in Chinese). (王鸣华，杨春龙，蒋木庚，世界农药, 2002, 24, 13.)

[8] Song, X.; Wang, S.; Tan, X.; Wang, Z.; Wang, Y. Chin. J. Org. Chem. 2007, 27, 72 (in Chinese).

(宋新建, 王胜, 谭小红, 王子云, 汪炎钢, 有机化学, 2007, 27, 72.)

[9] Peng, H.; He, H. W. Chin. J. Org. Chem. 2007, 27, 502 (in Chinese). (彭浩, 贺红武, 有机化学, 2007, 27, 502.)

[10] Chen, T.; Shen, P.; Li, Y. J.; He, H. W. J. Fluorine Chem. 2006, 127, 291.

[11] Cui, Z.; Li, Y.; Ling, Y.; Huang, J.; Cui, J.; Wang, R.; Yang, X. Eur. J. Med. Chem. 2010, 45, 5576. 